\title{
References
}

1 SotgiuG, Mauch V, Migliori GB, et al. Evidence-based, agreed-upon health priorities to remedy the tuberculosis patient's economic disaster. Eur Respir J 2014; 43: 1563-1566.

2 Voniatis C, Migliori GB, Voniatis M, et al. Tuberculosis elimination: dream or reality? The case of Cyprus. Eur Respir J 2014; 44: 543-546.

3 Diel R, Loddenkemper R, Zellweger JP, et al. Old ideas to innovate tuberculosis control: preventive treatment to achieve elimination. Eur Respir J 2013; 42: 785-801.

4 D'Ambrosio L, Dara M, Tadolini M, et al. TB Elimination: theory and practice in Europe. Eur Respir J 2014; 43: $1410-1420$.

5 Lönnroth K, Migliori GB, Abubakar I, et al. Towards tuberculosis elimination: an action framework for low-incidence countries. Eur Respir J 2015; 45: 928-952.

6 Esposito S, D'Ambrosio L, Tadolini M, et al. European Respiratory Society/World Health Organization Tuberculosis Consilium assistance with extensively drug-resistant tuberculosis management in a child: case study of compassionate delamanid use. Eur Respir J 2014; 44: 811-815.

7 Codecasa LR, Ciconali G, Mazzola E, et al. Managing an XDR-TB outbreak: the public health face of the medal. Eur Respir J 2015; 45: 292-294.

8 van der Werf MJ, Sandgren A, D'Ambrosio L, et al. The European Union standards for tuberculosis care: do they need an update? Eur Respir J 2014; 43: 933-942.

9 Falzon D, Jaramillo E, Schünemann HJ, et al. WHO guidelines for the programmatic management of drug-resistant tuberculosis: 2011 update. Eur Respir J 2011; 38: 516-528.

10 World Health Organization. Global Tuberculosis Report 2014. Document WHO/HTM/TB/2014.08. Geneva, World Health Organization, 2014.

11 Migliori GB, Sotgiu G, Gandhi NR, et al. Drug resistance beyond extensively drug-resistant tuberculosis: individual patient data meta-analysis. Eur Respir J 2013; 42: 169-179.

12 Falzon D, Gandhi N, Migliori GB, et al. Resistance to fluoroquinolones and second-line injectable drugs: impact on multidrug-resistant TB outcomes. Eur Respir J 2013; 42: 156-168.

13 Smit PW, Vasankari T, Aaltonen H, et al. Enhanced tuberculosis outbreak investigation using whole genome sequencing and IGRA. Eur Respir J 2015; 45: 276-279.

14 Abubakar I, Matthews T, Harmer D, et al. Assessing an outbreak of tuberculosis in an English college population. Eur Respir J 2011; 38: 976-978.

\section{Discrepant elevation of sIL-2R levels in sarcoidosis patients with renal insufficiency}

To the Editor:

Sarcoidosis is a systemic, granulomatous disease that can manifest in multiple organs [1]. Several biomarkers are used to assess disease activity and monitor response to therapy, including soluble interleukin-2 receptor (sIL-2R) and angiotensin-converting enzyme (ACE) [2]. sIL-2R was previously shown to correlate with the amount of $\mathrm{CD}^{+}$T-lymphocytes in bronchoalveolar lavage fluid [3]. Furthermore, it was shown that sIL-2R $>4000 \mathrm{pg} \cdot \mathrm{mL}^{-1}$ was a significant predictor of relapse after discontinuation of infliximab therapy and that sIL-2R is a suitable prognostic marker for disease progression $[4,5]$. We observed, however, that in a small number of patients with co-existing renal insufficiency, sIL-2R can be disproportionately high without marked signs of disease activity based on ${ }^{18}$ F-fluorodeoxyglucose positron emission tomography (FDG-PET), ACE and the clinical presentation. The aim of this pilot study is therefore to evaluate the influence of renal function on sIL-2R levels to determine if and how the marker can be used in sarcoidosis patients with renal insufficiency. In order to further illustrate the discrepancy, we first describe two cases.

The first case concerns a 56-year-old woman with pulmonary sarcoidosis, and a history of nephrotic syndrome and membranous glomerulonephritis resulting in moderate renal insufficiency (estimated glomerular filtration rate (eGFR) $50 \mathrm{~mL} \cdot \mathrm{min}^{-1}$ per $1.73 \mathrm{~m}^{2}$ ). ACE levels were repeatedly normal over a period of $>2$ years and no signs of sarcoidosis disease activity were present on the latest FDG-PET scan. However, sIL-2R levels remained highly elevated ( $>9000 \mathrm{pg} \cdot \mathrm{mL}^{-1}$; normal reference value $<3000 \mathrm{pg} \cdot \mathrm{mL}^{-1}$ ) despite treatment with infliximab.

The second case involves a 50-year-old man with sarcoidosis and a complicated history of stage $\mathrm{V}$ diabetic nephropathy, heart failure and various infections. The patient was treated with peritoneal dialysis. ACE was normal but sIL-2R levels were repeatedly extraordinarily high $\left(>50000 \mathrm{pg} \cdot \mathrm{mL}^{-1}\right)$ despite immunosuppressive 
treatment. FDG-PET scans showed only moderately active sarcoidosis in the pulmonary parenchyma and mediastinal and hilar lymph nodes. Although this patient still had fairly active sarcoidosis, potentially leading to an elevation in sIL-2R, the exceptionally high levels measured in this patient are rarely observed.

Both patients showed a higher than expected level of sIL-2R in concurrence with more or less severely impaired renal function. The observation of these and similar cases over the past few years led to the question of whether renal insufficiency results in elevated sIL-2R levels in sarcoidosis patients.

It has been described that sIL-2R is cleared by the kidneys [6], and an association between sIL-2R and renal function has been reported in a few small studies in other diseases [7, 8]. However, the implications for clinical use of sIL-2R as a disease activity marker in sarcoidosis patients with renal insufficiency remain unknown. Renal insufficiency in sarcoidosis patients may occur in cases of disease-associated hypercalcaemia or renal sarcoidosis, or result from comorbid conditions such as hypertension or diabetic nephropathy, as illustrated here.

To investigate the relation between serum sIL-2R levels and renal function, we performed a retrospective analysis in a cohort that was previously described by VorselaARs et al. [9]. This cohort consists of patients treated with methotrexate at St Antonius Hospital, Nieuwegein, which is a tertiary referral centre for interstitial lung disease in The Netherlands. All patients were treated between June 2004 and September 2011. Medical records were retrospectively reviewed for relevant demographic data, treatment and disease activity parameters. eGFR, calculated using the Modification of Diet in Renal Disease formula [10], was used as a marker for renal function. It was split into the following categories: insufficient, <60; sufficient-normal, 60-90; and sufficienthigh, $>90 \mathrm{~mL} \mathrm{~min}^{-1}$ per $1.73 \mathrm{~m}^{2}$. Importantly, sIL-2R levels are highly variable between patients depending on the amount of disease activity present, as described above. Hence, in order to be able to measure the pure effect of renal function on sIL-2R levels, we attempted to eliminate the factor of disease activity as much as possible by collecting all disease parameters after a period of 6 months of methotrexate treatment. Levels of sIL-2R were determined with DIACLONE ELISA Kit Human sIL-2R/sCD25 (Sanquin, Amsterdam, The Netherlands) according to manufacturer's instructions. Values $>3000 \mathrm{pg} \cdot \mathrm{mL}^{-1}$ are considered increased.

To evaluate the effect of renal function on sIL-2R levels, a multiple linear regression analysis was performed. Independent variables in the analysis included eGFR and ACE. As eGFR was divided into categories, it was recoded into the dummy variables eGFR $<60$ and $60-90 \mathrm{~mL} \cdot \mathrm{min}^{-1}$ per $1.73 \mathrm{~m}^{2}$, where eGFR $>90 \mathrm{~mL} \cdot \mathrm{min}^{-1}$ per $1.73 \mathrm{~m}^{2}$ was used as a reference category. ACE (divided into normal $\left(<70 \mathrm{U} \cdot \mathrm{L}^{-1}\right.$ ) and high $\left(>70 \mathrm{U} \cdot \mathrm{L}^{-1}\right)$ ) was merely introduced into the model as another activity marker to adjust for remaining disease activity, for reasons described earlier. The study was approved by the local institutional review board (St Antonius Hospital, Nieuwegein, The Netherlands) with registration number LTME/ Z-12.05 and acronym METHVERAZ.

In 86 patients out of the initial 145, data on renal function and disease activity markers at corresponding time-points were available. Of these, $63 \%$ were male and the treatment indication was related to pulmonary involvement in $66 \%$ of patients. In total, seven patients were classified as having renal insufficiency by an eGFR $<60 \mathrm{~mL} \cdot \mathrm{min}^{-1}$ per $1.73 \mathrm{~m}^{2}$.

A significant association was found between sIL-2R and insufficient eGFR of $<60(p=0.001)$ relative to sufficient-high eGFR of $>90 \mathrm{~mL} \cdot \mathrm{min}^{-1}$ per $1.73 \mathrm{~m}^{2}$. Importantly, no association was found between sIL-2R and sufficient-normal eGFR of 60-90 relative to a sufficient-high eGFR of $>90 \mathrm{~mL} \cdot \mathrm{min}^{-1}$ per $1.73 \mathrm{~m}^{2}$ ( $p=0.992$ ). Both results were adjusted for disease activity by ACE. Because of the small sample size of the eGFR $<60 \mathrm{~mL} \cdot \mathrm{min}^{-1}$ per $1.73 \mathrm{~m}^{2}$ group and the resulting imperfect normal distribution, we also performed a nonparametric Kruskal-Wallis test, which gave similar results. Results are shown in figure 1.

These findings suggest that renal function impairment may indeed have a significant influence on sIL-2R levels in patients with sarcoidosis. Importantly, it also shows that sIL-2R levels do not correlate with normal renal function. Thus, in patients with renal insufficiency, sIL-2R levels may rise and probably also reflect accumulation through impaired renal clearance, instead of just sarcoidosis disease activity. This may lead to an overestimation of actual disease activity in this specific patient group. Therefore, we suggest caution regarding the interpretation of sIL-2R levels when renal function is impaired.

Theoretically, not only impaired clearance of sIL-2R but also increased production may lead to a higher than expected level of sIL-2R [11]. In patients with end-stage renal disease, T-cells appear to be pre-activated and, therefore, express more interleukin-2 receptor on their membrane. As a result, more shed sIL-2R appears in the blood. High sIL-2R levels in patients with stage $\mathrm{V}$ renal failure could thus be both the result of impaired clearance and increased production. In addition, it is worth mentioning that patients with chronic renal failure who are on maintenance dialysis show higher sIL-2R levels than undialysed patients $[11,12]$.

This study is limited by the small sample size and the retrospective design. In addition, this was a pilot study in an existing cohort. However, since renal insufficiency is a contraindication to the prescription of 


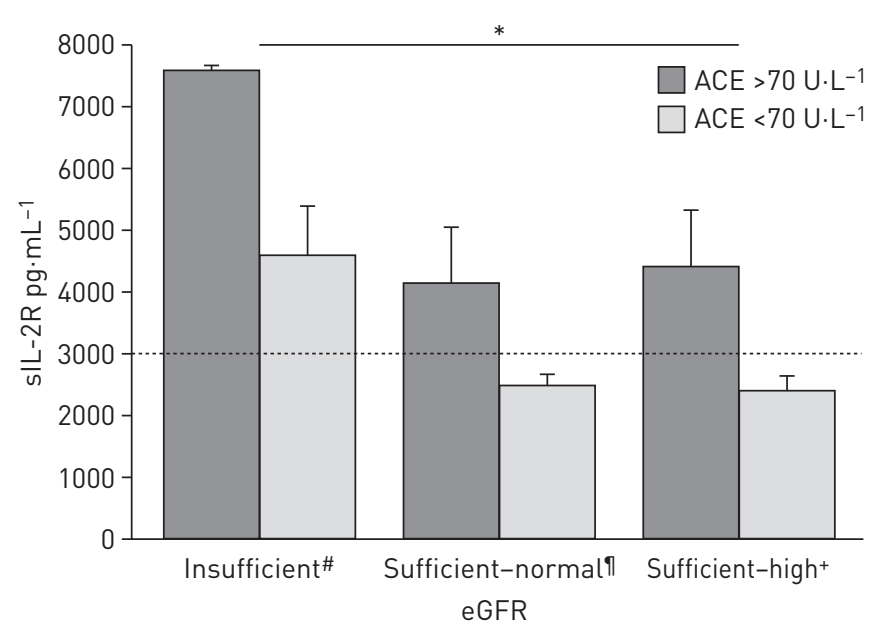

FIGURE 1 Mean \pm SEM levels of soluble interleukin-2 receptor (sIL-2R) are displayed per category of estimated glomerular filtration rate (eGFR). These categories were subsequently split into normal angiotension-converting enzyme (ACE) $\left(<70 \mathrm{U} \cdot \mathrm{L}^{-1}\right)$, representing low disease activity, and high ACE $\left(>70 \mathrm{U} \cdot \mathrm{L}^{-1}\right)$, representing high disease activity. The mean levels of sIL-2R are considerably higher in the patient group with insufficient eGFR, reflecting accumulation. The dotted line indicates the normal reference value of sIL-2R $\left(<3000 \mathrm{pg} \cdot \mathrm{mL}^{-1}\right) .{ }^{\#}:<60 \mathrm{~mL} \cdot \mathrm{min}^{-1}$ per $1.73 \mathrm{~m}^{2} ;{ }^{\text {ๆ }}: 60-90 \mathrm{~mL} \cdot \mathrm{min}^{-1}$ per $1.73 \mathrm{~m}^{2} ;^{+}:>90 \mathrm{~mL} \cdot \mathrm{min}^{-1}$ per $1.73 \mathrm{~m}^{2}$.

methotrexate, this cohort might underestimate the occurrence of renal insufficiency in the general sarcoidosis patient population. The effect will possibly be more substantial when a larger, more representative cohort is evaluated.

In conclusion, this pilot study shows that renal insufficiency has a significant influence on sIL-2R levels in sarcoidosis patients. Assessment of renal function when interpreting sIL-2R values is therefore warranted. Although this study only involved sarcoidosis patients, the results may likely be extended to other diseases in which sIL-2R is used as a biomarker for disease evaluation. insufficiency http://ow.ly/ICVrY

Anouk Verwoerd ${ }^{1}$, Adriane D.M. Vorselaars ${ }^{1}$, Coline H.M. van Moorsel ${ }^{1,2}$, Willem Jan W. Bos ${ }^{3}$, Heleen van VelzenBlad $^{4}$ and Jan C. Grutters ${ }^{1,2}$

${ }^{1}$ Dept of Pulmonology, Interstitial Lung Diseases Centre of Excellence, St Antonius Hospital, Nieuwegein, The Netherlands. ${ }^{2}$ Division of Heart and Lungs, University Medical Centre Utrecht, Utrecht, The Netherlands. ${ }^{3}$ Dept of Internal Medicine, St Antonius Hospital, Nieuwegein, The Netherlands. ${ }^{4}$ Dept of Medical Microbiology and Immunology, St Antonius Hospital, Nieuwegein, The Netherlands.

Correspondence: Anouk Verwoerd, Interstitial Lung Diseases Centre of Excellence, Dept of Pulmonology, St Antonius Hospital, Koekoekslaan 1, 3435 CM Nieuwegein, The Netherlands. E-mail: a.verwoerd@antoniusziekenhuis.nl

Received: Jan 132015 | Accepted after revision: Jan 252015 | First published online: March 052015

Conflict of interest: None declared.

Acknowledgements: We thank Ellen Tromp, statistician at St Antonius Hospital, Nieuwegein, The Netherlands, for her statistical assistance.

\section{References}

Iannuzzi MC, Rybicki BA, Teirstein AS. Sarcoidosis. N Engl J Med 2007; 357: 2153-2165.

2 Vorselaars AD, van Moorsel CH, Zanen $\mathrm{P}$, et al. ACE and sIL-2R correlate with lung function improvement in sarcoidosis during methotrexate therapy. Respir Med 2015; 109: 279-285.

3 Grutters JC, Fellrath JM, Mulder L, et al. Serum soluble interleukin-2 receptor measurement in patients with sarcoidosis: a clinical evaluation. Chest 2003; 124: 186-195.

4 Vorselaars AD, Verwoerd A, van Moorsel CH, et al. Prediction of relapse after discontinuation of infliximab therapy in severe sarcoidosis. Eur Respir J 2014; 43: 602-609.

5 Ziegenhagen MW, Benner UK, Zissel G, et al. Sarcoidosis: TNF- $\alpha$ release from alveolar macrophages and serum level of sIL-2R are prognostic markers. Am J Respir Crit Care Med 1997; 156: 1586-1592.

6 Junghans RP, Waldmann TA. Metabolism of Tac (IL2R $\alpha$ ): physiology of cell surface shedding and renal catabolism, and suppression of catabolism by antibody binding. J Exp Med 1996; 183: 1587-1602. 
7 Seidler S, Zimmermann HW, Weiskirchen R, et al. Elevated circulating soluble interleukin-2 receptor in patients with chronic liver diseases is associated with non-classical monocytes. BMC Gastroenterol 2012; 12: 38.

8 Nässberger L, Sturfelt G, Thysell H. Serum levels of the soluble interleukin-2 receptor are dependent on the kidney function. Am J Nephrol 1992; 12: 401-405.

9 Vorselaars AD, Wuyts WA, Vorselaars VM, et al. Methotrexate $v s$ azathioprine in second-line therapy of sarcoidosis. Chest 2013; 144: 805-812.

10 Levey AS, Coresh J, Greene T, et al. Using standardized serum creatinine values in the modification of diet in renal disease study equation for estimating glomerular filtration rate. Ann Intern Med 2006; 145: $247-254$.

11 Descamps-Latscha B, Herbelin A, Nguyen AT, et al. Balance between IL-1 $\beta$, TNF- $\alpha$, and their specific inhibitors in chronic renal failure and maintenance dialysis. Relationships with activation markers of $\mathrm{T}$ cells, $\mathrm{B}$ cells and monocytes. J Immunol 1995; 154: 882-892.

12 Shu KH, Lu YS, Cheng CH, et al. Soluble interleukin 2 receptor in dialyzed patients. Artif Organs 1998; 22: 142-144.

\section{0-year long-term survival of a metastatic EGFR-mutated nonsmall cell lung cancer patient}

\section{To the Editor:}

10 years ago, the US Food and Drug Administration approved erlotinib in the second and third line settings for unselected advanced nonsmall cell lung cancer (NSCLC) patients. Activating EGFR mutations were discovered by analysing patient subgroups who responded to oral, first-generation, single-target reversible EGFR tyrosine kinase inhibitor (TKI). Nine randomised phase III chemotherapy-controlled trials in EGFR-mutant NSCLC naive patients showed the superiority of gefitinib, erlotinib and afatinib, respectively, in terms of response rate and progression-free survival. Response rate is in the range of $70 \%$ and progression-free survival about 1 year. Exon 19 in-frame deletions represent about $45 \%$ of overall EGFR mutations and half of the sensitising ones [1]. New generation targeted therapies are currently under clinical development. However, treatment sequence is still debated after a first line of EGFR TKI regimen in these molecularly selected patients. We herein report a case of a metastatic EGFR-mutated lung adenocarcinoma patient who achieved a prolonged survival of 10 years through multiple surgical and medical treatments (fig. 1).

Our female patient was 60 years old when a chronic cough led to the diagnosis of a right lower lobe adenocarcinoma in January 2005. She had smoked five to six cigarillos per day from the age of 53 to 58 years, was treated for hypertension and had undergone a hysterectomy in 1999 for personal convenience. She presented an initial pericardial effusion with a positive cytology. The tumour was then classified as stage IV (cT4N1pM1a), according to the current seventh TNM classification. Thus, she was treated with a six-cycle platinum-based chemotherapy doublet. The tumour progressed in November 2006 and new biopsies revealed an E746 A750 deletion in exon 19 of the EGFR gene. Neratinib was then unsuccessfully administered for 3 months with grade 2 diarrhoea and grade 1 skin ulceration of the index finger. The tumour progressed through three cycles of pemetrexed. It was proposed that our patient join a phase 1 trial testing an oral signal-transduction inhibitor with both anti-angiogenic (VEGFR1-3 inhibition) and anti-HER functions (HER1, HER2, HER4): BMS690514 [2]. She presented grade 1 facial folliculitis, grade 2 diarrhoea and grade 3 hypertension, despite an association of angiotensin-converting enzyme inhibitor and thiazide diuretic. Therefore, the drug dose was decreased by 25\%. BMS690514 was interrupted after 1 month because of a Tako-Tsubo cardiomyopathy and restarted with half a dose. After 15 months of treatment, the patient underwent an extended surgical procedure in a context of remarkable partial response. Tumour residues were found in mediastinal adenopathies which led to local irradiation. BMS690514 was discontinued after a 5-month adjuvant therapy. Interestingly, post-operative molecular analyses did not reveal any EGFR mutation but a chromosome 7 polysomy. 1 year later the tumour relapsed in all lung parenchyma. Gefitinib was unsuccessfully administered for 1 month and switched for a sixth line prescription of $85 \%$ full-dose erlotinib (because of grade 2 skin toxicity). The disease remained stable for 3 years in a context of manageable grade 2 skin toxicities and one resected skin basal cell carcinoma. In October 2013, the patient was enrolled on the AZD 9291 phase 1 trial testing this oral daily irreversible EGFR inhibitor. After 1 year, she still presents an excellent partial response with excellent physical condition and normal daily activities. 